\title{
Die Gründungsgeschichte des Verkehrs- und Baumuseums in Berlin (1879-1906) im Schatten des Deutschen Museums
}

\author{
VON ECKHARD SCHINKEL
}

\begin{abstract}
Überblick
Das 1906 in Berlin eröffnete Verkehrs- und Baumuseum griff auf eine Idee für ein preußisches Eisenbahnmuseum aus dem Jahr 1879 zurück. Die Gründungsinitiative des Ministeriums der öffentlichen Arbeiten 1902/03 folgte weniger einem inhaltlichen Konzept als innen- und außenpolitischen Impulsen. Nach innen diente das Museum der Demonstration institutioneller Identität, da das Ministerium der öffentlichen Arbeiten mehrfach in seiner Existenzberechtigung politisch infrage gestellt worden war. Nach außen setzte das Museum staatspolitisch ein Zeichen preußischer Identität, da es mit der Sicherung preußischen Kulturguts der reichsweiten intensiven Sammlungstätigkeit für ein nationales „Museum von Meisterwerken der Naturwissenschaft und Technik" in München begegnete. Um Zustimmung zu erhalten, musste das Berliner Museumsprojekt dem preußischen König ohne Anlass zum Konflikt präsentiert werden, da Wilhelm II. als deutscher Kaiser das Deutsche Museum in München mit großer Anteilnahme unterstützte. Die Anfänge der Museen in München und in Berlin waren so von Konkurrenz und Gleichklang geprägt.
\end{abstract}

\begin{abstract}
In 1906, the Museum for Traffic and Public Buildings opened in Berlin. The Prussian Ministry for Public Works launched the initiative for this museum project in 1902-03. Though it drew upon earlier plans for a Prussian railway museum, the overall motivation for the construction of the new traffic and buildings museum had little to do with its projected content. Instead, it had to do with the prevailing winds of domestic and foreign affairs. Internally, the new museum was to serve as a demonstration of institutional identity and purpose for the Ministry of Public Works, for the ministry's raison d'être was at the time the subject of public debate. On the level of the national policies of the Kaiserreich, the new museum was meant both to celebrate Prussian identity and to protect Prussian cultural assets from the intensive collection efforts undertaken by Oskar von Miller for his own museum project, the Munichbased national „Museum von Meisterwerken der Naturwissenschaft und Technik“ (Deutsches Museum). Plans for the Berlin traffic and building museum were presented to Wilhelm II as Prussian king for royal approval, but because
\end{abstract}


Wilhelm II as German Kaiser strongly supported the Deutsche Museum project, all notions of open conflict between the two projects were swept under the rug. The early relationship between the Berlin and Munich museums thus was characterized by public consonance and behind-the-scenes competition.

\section{Im Schatten des Deutschen Museums}

Ein Mittel der Politik ist ein Museum, noch bevor es eröffnet wird. ${ }^{1}$ Kontroversen zur Existenzberechtigung, zur Sicherung, zur Positionierung und Etablierung in einem politisch-gesellschaftlichen Umfeld, Konkurrenzen und Abgrenzungen auf besetzten Feldern und nicht zuletzt die Stellung und Persönlichkeit der Akteure bilden ein dichtes wie fragiles Beziehungsgeflecht. Diese Beziehungen beeinflussen die Formierung eines Museums und wirken sich auf das Konzept, auf die Sammlungspolitik, auf seine gesamte Erscheinungsweise aus. Deshalb ist die Geschichte der Museen, insbesondere die Geschichte der Museumsgründungen in der Regel „keine friedliche Geschichte“.2

Im Blick zurück auf eine erfolgreiche Gründung, besonders anlässlich von Jubiläen, und in den Selbstdarstellungen einzelner Museen traten und treten diese Vorgänge nicht selten in den Hintergrund; hier werden sie verdrängt, dort einfach vergessen. Das Musterbeispiel für derartige Umbesetzungen ist die Geschichtsschreibung zum Deutschen Museum in München. Über mehrere Jahrzehnte hinweg überformten die Autorität, das Charisma und der Erfolg seines Gründers Oskar von Miller die komplexe Entstehungsgeschichte des Museums. ${ }^{3}$ Eng verbunden mit diesem Erfolg war es nicht

1 Ein besonderer Dank für ihre Unterstützung gilt den Kolleginnen und Kollegen aus dem Deutschen Technikmuseum, Herrn Böndel, Herrn Nußbaum, Herrn Schmalfuß, Herrn Brennigk, aus dem Geheimen Staatsarchiv Preußischer Kulturbesitz Frau Krause, Frau Drechsler und Herrn Strecke. Hilfreich waren die Veröffentlichungen von Alfred Gottwaldt, Holger Steinle, Verkehrs- und Baumuseum Berlin: der Hamburger Bahnhof, Berlin 1984; Hanno Möbius, Vierhundert Jahre technische Sammlungen in Berlin, Berlin 1983; Uwe Nußbaum, Eisenbahnmodelle. Schätze aus dem Verkehrs- und Baumuseum, Berlin 1998; unveröffentlicht: Martin Brennigk, Das Verkehrs- und Baumuseum Berlin. Rekonstruktion der Bau- und Sammlungsgeschichte des Verkehrs- und Baumuseums im Hamburger Bahnhof, Diplomarbeit FH Technik und Wirtschaft Berlin 1999; Michael Kamp, Das Museum als Ort der Politik. Münchner Museen im 19. Jahrhundert, Dissertation München 2002. [Veröffentlichung im Internet: http://edoc.ub.uni-muenchen.de/archive/ 00004080]

2 Analog zu der Feststellung von Wolf Lepenies, „daß die Geschichte der Wissenschaften keine einfache und (in der Regel) keine friedliche Geschichte ist. Geprägt wird sie vielmehr von der Auseinandersetzung nationaler Traditionen und vom Streit individueller Forschertemperamente, von Prioritätsansprüchen und Plagiatsvorwürfen, vom Kampf um materielle und geistige Ressourcen und vom Versuch verschiedener Disziplinen, über die wissenschaftliche Erkenntnis hinaus lebensweltliche Orientierungsleistungen zu erbringen“, vgl. Wolfgang Lepenies, Gefährliche Wahlverwandtschaften. Essays zur Wissenschaftsgeschichte, Stuttgart 1989, S. 5.

3 Wilhelm Füßl, Oskar von Miller. 1885-1934. Eine Biographie, München 2005 (ohne Bezug zum Verkehrs- und Baumuseum). 
nur als Institution zum Modell geworden. Ihm war zudem gelungen, was Wilhelm Füß1 als „Konstruktion technischer Kultur“ bezeichnet hat. ${ }^{4}$ Damit verfestige, vervielfachte und popularisierte es Leitbilder von der technischen Entwicklung, von den Entdecker- und Erfinderpersönlichkeiten, von ,technischem Heroenkult“ (Wolfhard Weber) - „Männer machen Geschichte“ - , von ausgesuchten Einzelobjekten als den „Meisterwerken“, denen ihr geschichtlicher Gehalt, die Zusammenhänge ihres Entstehens abhanden gekommen waren. Nicht zuletzt avancierte die Institution Deutsches Museum damit selbst ein Stück weit zum Meistermacher.

Die populären Leitbilder wirken in vielen Darstellungen zu Technik, Industrie und Verkehr bis heute nach. So mag die Vielzahl der Publikationen zum Deutschen Museum auch ein Indiz für die Wirkmächtigkeit dieses Über-Museums sein und dafür, um wie viel schwieriger die Korrektur jener Leitbilder gegenüber ihrer Etablierung ist. Mit der Veröffentlichung des Projekts für ein nationales „Museum von Meisterwerken der Naturwissenschaften und Technik" traten andere Projekte in den Hintergrund der öffentlichen Wahrnehmung oder gar nicht erst aus ihm heraus, so auch das 1906 eröffnete Verkehrs- und Baumuseum in Berlin. Die Gründe dafür sind vielfältig und zusätzlich Anlass, nach dem Spannungsfeld und den Konfliktszenarien in seiner Gründungsphase und ihren Einflüssen auf die Museumsentwicklung zu fragen.

\section{Chronik und Vorgeschichte}

Im Zuge der technischen und betrieblichen Rationalisierung wurden 1879 in Preußen die Privateisenbahnen verstaatlicht. Die bürokratische Modernisierung zielte auf eine „schärfere [...] Zentralisierung“.5 Das Eisenbahnwesen, manchmal als „Staat im Staat" apostrophiert und die wichtigste Einnahmequelle des preußischen Staatshaushalts, wurde dem Ministerium der öffentlichen Arbeiten unterstellt. Gleichwohl hatten die „Eisenbahner“ einen hervorgehobenen Status, zumal die Minister nicht selten selbst aus dem Eisenbahnfach kamen und über Eisenbahnerfahrungen verfügten. Die Idee für ein Eisenbahnmuseum tauchte, ähnlich wie in Bayern, ${ }^{6}$ schon vor der Verstaatlichung auf. Ein „Ursprung“ der Idee ließ sich bisher nicht ermitteln. Überliefert ist ein Brief des Ministers der öffentlichen Arbeiten in Preußen von Maybach ${ }^{7}$

4 Ders., Konstruktion technischer Kultur. Sammlungspolitik des Deutschen Museums in den Aufbaujahren 1903-1909, in: Ulf Hashagen, Oskar Blumtritt u. Helmuth Trischler (Hg.), Circa 1903. Artefakte in der Gründungszeit des Deutschen Museums, München 2003, S. 32-53.

5 Fr. E., Der Wechsel im preuss. Ministerium der öffentlichen Arbeiten, in: Deutsche Bauzeitung 52, 28. Juni 1902, S. 333-336, hier S. 333.

6 Olaf Hartung, Museen des Industrialismus. Formen bürgerlicher Geschichtskultur am Beispiel des Bayerischen Verkehrsmuseums und des Deutschen Bergbaumuseums, Köln, Weimar u. Berlin 2007, S. 89ff.

7 Brief des Ministers der öffentlichen Arbeiten an die Königl. Direction der Niederschlesisch Märkischen Eisenbahn, Berlin 27. Mai 1879, Berlin, Deutsches Technikmuseum, Historisches Archiv, Archiv-Bestand Verkehrs- und Baumuseum (= HH): HH 15. Eine kriti- 
aus dem Jahr 1879 an die „Königl. Direction der Niederschlesisch Märkischen Eisenbahn". Vermutlich wurden gleichlautende Briefe an die anderen Eisenbahndirektionen in Preußen gerichtet. Über die vorgängige Entwicklung, als deren Ergebnis der Brief zu lesen ist, ist nichts bekannt. Ziel der geplanten Zusammenführung der zahlreichen Modelle aus der „Bau- und Gewerbe-Akademie" in Berlin mit den Modellen und Probestücken in der Eisenbahnverwaltung sei,

„daß die Entwicklung des Eisenbahnwesens in seinen verschiedenen Stadien daran verfolgt und studirt werden kann. Es würde hiermit der Grund zu einem demnächst weiter auszubauenden Eisenbahn-Museum, ähnlich wie neuerdings zur Darstellung des Entwicklungsganges im Postwesen ein Post-Museum gegründet worden ist, gelegt werden können und hierbei der doppelte Zweck ins Auge zu fassen sein, sowohl dem Publikum Gelegenheit zu Informationen über die Geschichte und Technik des Eisenbahnwesens zu geben, als auch den Studirenden der technischen Hochschule durch übersichtliche Anordnung der Lehrmittel eine Erleichterung des Studiums zu gewähren". ${ }^{8}$

Der Minister der geistlichen, Unterrichts- und Medizinal-Angelegenheiten, so von Maybach, unterstütze das Museumsprojekt. Hinter der Darstellung der „Entwicklung des Eisenbahnwesens in seinen verschiedenen Stadien“ steht die Vorstellung von einer Fortschritts- als Erfolgsgeschichte. Das Eisenbahnmuseum in Berlin sollte ein Repräsentant der modernen preußischen Eisenbahn werden. Zur weiteren Entwicklung hält der Rechenschaftsbericht der Museumsverwaltung aus dem Jahr 1913 fest:

„Anfangs wurde die Verwirklichung in Gestalt der Angliederung eines Eisenbahn-Museums an die Technische Hochschule zu Charlottenburg gesucht. Dieser Gedanke, welcher seit 1882 mit der Errichtung eines Curatoriums des Eisenbahn-Museums und der Überweisung einer größeren Anzahl von Modellen und Lehrgegenständen aus dem Gebiete des Lokomotivwesens greifbare Gestalt angenommen hatte, musste bereits im Jahre 1884 wegen Raummangels sistiert werden. Das Curatorium löste sich auf, die Gegenstände wurden den LehrmittelSammlungen der Abteilungen II und III der Hochschule überwiesen. Der Gedanke selbst war damit aber nicht eingeschlafen; die in Verfolg des eingeleiteten Beschaffungsgedankens eingehenden Sammlungs-Gegenstände der preußischen Eisenbahndirektionen, wie von Privatfirmen, besonders des Eisenbahnwesens wurden nunmehr in einigen für diese Zwecke eingerichtete Räumen des Ministeriums der öffentlichen Arbeiten vorübergehend bis zur endgiltigen Schaffung würdiger Räume untergebracht." ${ }^{\text {99 }}$

sche Auseinandersetzung mit der Arbeit des Ministeriums veröffentlichte die Deutsche Bauzeitung 1891, S. 344-347, S. 370f. u. S. 401f. unter dem Titel „Das preussische Ministerium der öffentlichen Arbeiten unter Staats-Minister v. Maybach“.

8 Ebd.

9 Masch.schr. Rechenschaftsbericht der Museumsverwaltung vom März 1913, S. 3, Berlin, DTM: HH 99. 
Das Eisenbahnmuseum in Berlin wurde damals nicht verwirklicht. Es fehlte, wie es heißt, an geeigneten Räumen. 1904 griff das preußische Ministerium der öffentlichen Arbeiten die Museumsidee offiziell und mit einer entscheidenden Modifikation wieder auf. Konkrete Vorbereitungen waren vorausgegangen, ,,indem im Verein mit den einschlägigen Ministerien Schritte eingeleitet wurden, welche auf eine Nutzbarmachung der Gebäude des außer Betrieb gestellten Hamburger Bahnhofes hinausgingen“. ${ }^{10}$ Über diese „Schritte“ ist nichts weiter bekannt. Doch statt für ein Eisenbahnmuseum legte das Ministerium der öffentlichen Arbeiten den Plan für ein ,Verkehrs- und Baumuseum" vor. Die drei Abteilungen des Ministeriums: Eisenbahn, Wasserbau, Hochbau sollten darin vertreten sein.

Im Januar 1905 beantragte der Staatsminister der öffentlichen Arbeiten Hermann von Budde 650.000 Mark aus dem preußischen Staatshaushalt. Für diesen Betrag sollte der Hamburger Bahnhof umgebaut, sollten Sammlungen zusammengestellt, Modelle gebaut und das gesamte Haus eingerichtet werden. Unter Minister von Budde und unter seinem Nachfolger Paul Justin von Breitenbach wurde das Museum in knapp zwei Jahren aufgebaut und am 14. Dezember 1906 eröffnet.

Entscheidendes bleibt in dieser Chronik der Ereignisse ausgeblendet. Wodurch wurde der Halbschlaf des Museumsgedankens beendet? Hinweise dazu finden sich weder in der unmittelbaren Aktenüberlieferung zum Verkehrs- und Baumuseum, noch in den späteren Darstellungen zum Verkehrs- und Baumuseum. Dennoch „kam“ es nicht ohne Grund „zur Einrichtung eines Bau-

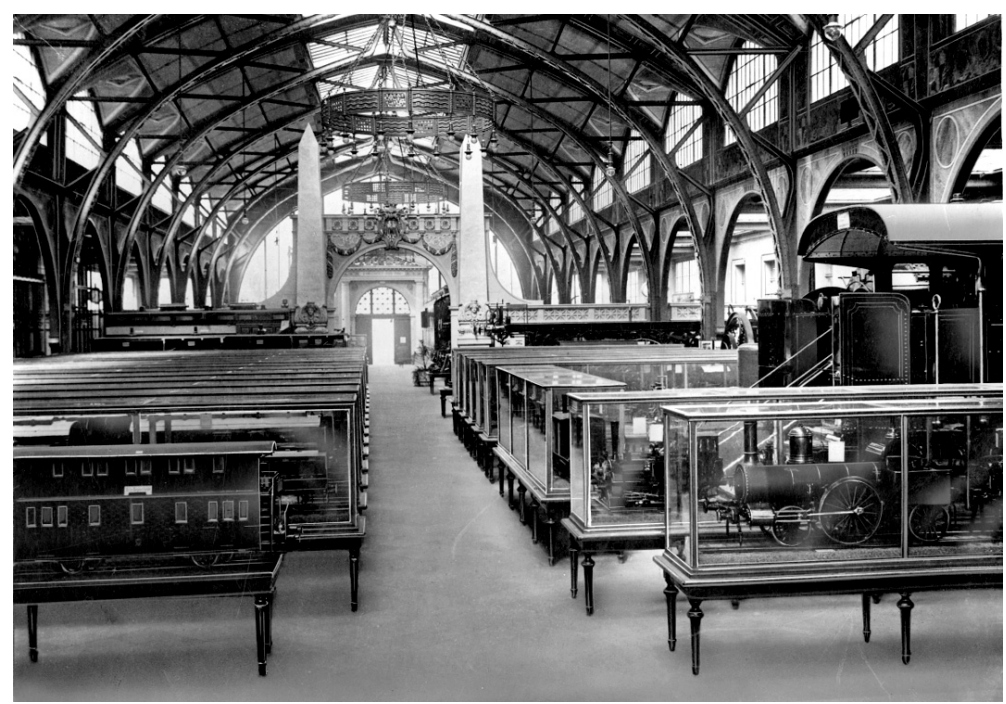

Abb. 1: Der zentrale Saal mit der Eisenbahnsammlung im neu eröffneten Verkehrs- und Baumuseum (1906). Foto: Deutsches Technikmuseum, Berlin.

10 Ebd., S. 3f. 
und Verkehrsmuseum [sic] ${ }^{\text {“. }}{ }^{11}$ Auch die Formulierung aus der Festrede Minister von Breitenbachs, ,dass mit der Eröffnung ein seit drei Jahrzehnten lang gehegter Wunsch in Erfüllung gehe“", verschleierte die historischen Tatsachen. ${ }^{12}$ Im Allgemeinen gilt 1904/5 als das Einsatz-Jahr. ${ }^{13} 1905$ schrieb der Minister der öffentlichen Arbeiten von Budde den in den Darstellungen zur Gründung des Verkehrs- und Baumuseums immer wieder zitierten Brief an den Kaiser und König von Preußen, Wilhelm II., mit der Bitte um Einverständnis zur „Herstellung eines Verkehrs- und Baumuseums in Berlin“:

„In dem ehemaligen Empfangsgebäude der Hamburger Bahn zu Berlin sollen Räume zur Unterbringung von Modellen, Probestücken, Plänen usw. aus dem Gebiete des Verkehrs- und Bauwesens eingerichtet werden. Diese bereits jetzt in großer Zahl vorhandenen Gegenstände befinden sich z.Zt. an den verschiedensten Stellen zerstreut und meist in unzulänglichen und zur Aufbewahrung wenig geeigneten Räumen. Das neue Museum soll der allgemeinen Besichtigung zugänglich gemacht werden und dazu dienen, für die Geschichte und Technik des Eisenbahn- und Bauwesens und für die Einrichtungen des Verkehrswesens in weiteren Kreisen des Volkes Verständnis und Interesse zu erwecken; im besonderen ist beabsichtigt, durch Reichhaltigkeit und übersichtliche Anordnung der Sammlungen Fachleuten, Beamten und Studierenden in mannigfacher Beziehung Belehrendes zu bieten und ihre Kenntnisse zu fördern. Die Gesamtkosten der zur Einrichtung der Räume erforderlichen Bauausführungen sind mit $650000 \mathrm{M}$. auf den Etat übernommen." ${ }^{\text {"14 }}$

Ein Jahr vorher, im Januar 1904, hatte von Budde eine Kommission zur Vorbereitung des Museums einberufen. Die Vorbereitungen fallen demnach ins Jahr 1903, wie der Rechenschaftsbericht aus dem Jahr 1913 bestätigt. Stimmt die Angabe im Rechenschaftsbericht, wonach der Museumsgedanke noch unter Minister von Thielen ,neue Gestalt“ annahm, können die Vorbereitungen schon 1902, vor von Thielens Rücktritt im selben Jahr begonnen haben. ${ }^{15}$

Zwei Impulse haben den Ausschlag für die Wiederaufnahme und für die Umformulierung der Museumsgründungsidee in den Jahren 1902/3 gegeben: ein innenpolitischer und ein außenpolitischer Impuls.

11 Wolfhard Weber, Die Gründung technischer Museen in Deutschland im 20. Jahrhundert, in: Museumskunde 56, 1991, S. 82-93, hier S. 83 und Möbius (wie Anm. 1), S. 98.

12 Ebd., S. 4; Einen Beleg dafür, dass die Museumsgründung auf Initiative des Ministerialdirektor Althoff zurückzuführen sei, wie Gundel und Gert Mattenklott ausführen, habe ich nicht finden können, vgl. Gert u. Gundel Mattenklott, Ausstellungsbahnhof Berlin, in: Ausstellungs-Katalog Berlin 1987. Berlin Transit. Eine Stadt als Station, Berlin 1987, S. 283-288.

13 Brennigk (wie Anm. 1), S. 31f.; Möbius (wie Anm. 1), S. 98.

14 An des Kaisers und Königs Majestät, Berlin, den 20. Januar 1905, Berlin, GStAPK: I. HA Rep. 93 B, Nr. 3839/9, B1. 122f.

15 Rechenschaftsbericht (wie Anm. 9), S. 3. Offenbar versehentlich wird „1903“ anstelle von 1902 als Jahr von von Thielens Rücktritt angegeben. 


\subsection{Demonstration institutioneller Identität}

Der innenpolitische Impuls kam aus dem preußischen Abgeordnetenhaus. Aus dem Lager der Agrarier wurde zum wiederholten Mal ein Vorschlag zur Diskussion gebracht, hinter dem nichts weniger stand als die Umstrukturierung, die Verkleinerung oder sogar die Abschaffung des Ministeriums der öffentlichen Arbeiten. Bereits 1895 hatte der Landwirtschaftsminister Freiherr von Hammerstein einen Vorschlag zur „Abtrennung der Bauverwaltung vom Ministerium der öffentlichen Arbeiten" unterbreitet; auch innerhalb der Staatseisenbahnverwaltungen gab es Stimmen für diese Trennung von der als Konkurrenz angesehenen Wasserbauverwaltung. ${ }^{16}$ Zur gleichen Zeit, im Anschluss an seine Kritik am Hochwasserschutz in Preußen forderte auch Wilhelm II. eine Neuausrichtung des Ministeriums. Es sei in ein Eisenbahnministerium umzuwandeln; der Hochwasserschutz solle dem Landwirtschaftsministerium zugeordnet werden. ${ }^{17}$ Nach der Jahrhundertwende forderten die konservativen Agrarier erneut die Unterstellung der Wasserbauabteilung des Ministeriums der öffentlichen Arbeiten unter das Landwirtschaftsministerium, womit ihr Einfluss auf die Wasserstraßenpolitik erheblich gewachsen wäre. ${ }^{18}$

Der politische Hintergrund für die Vorstöße der konservativen Agrarier war die bereits zweimal gescheiterte „Wasserwirtschaftliche Vorlage“ mit einem der ambitioniertesten innenpolitischen Projekte der preußischen Staatsregierung: dem Mittellandkanal. ${ }^{19}$ Geplant war eine Wasserstraßenverbindung zwischen den Flüssen, Rhein, Weser, Elbe und Oder. Gegen viele Widerstände förderte die Regierung den Aufbau der Binnenschifffahrt als zweites Transportsystem neben der Eisenbahn für die Massengüter Kohle und Eisen. Die Agrarier befürchteten, dass auf diesem Weg billiges Importgetreide nach Preußen kommen und ihren Produkten Konkurrenz machen würde. Als König von Preußen hatte sich Kaiser Wilhelm II. hinter das Mittellandkanalprojekt gestellt. Als die Kanalvorlage 1902 erneut abgelehnt wurde, reichte der amtierende Minister der öffentlichen Arbeiten von Thielen seinen Rücktritt ein. Sein Nachfolger wurde der Generalmajor a.D. von Budde. ${ }^{20}$

Das Ministerium der öffentlichen Arbeiten - jetzt unter von Budde wies das Ansinnen der Agrarier ab und ging nun seinerseits in die Offensive und zwar mit einem programmatischen Bekenntnis:

16 Zeitschrift für Binnenschifffahrt, H. 8, 1895, S. 235 und H. 10, 1895, S. 291; rückblickend dazu: Fr. E. (wie Anm. 5), S. 333.

17 S. dazu Wolfgang König, Wilhelm II. und die Moderne. Der Kaiser und die technischindustrielle Welt, Paderborn 2007, S. 99.

18 Fr. E., Das Ministerium der öffentlichen Arbeiten und die Techniker in den diesjährigen Verhandlungen des preussischen Abgeordnetenhauses, in: Deutsche Bauzeitung, 22. April 1903, S. 207f. u. 218ff.; hier S. 207.

19 Eckhard Schinkel, Der Mittellandkanal. Neue Beiträge zur Geschichte, in: 100 Jahre Mittellandkanal. Tradition und Innovation (Supplement „Binnenschifffahrt“), Nr. 4, 2005, S. 5 13.

20 Fr. E. (wie Anm. 5), S. 333. 
„An sich ist in einer Zeit, in welcher die Technik eine solche Rolle spielt, wie heute, sicher nicht ganz ohne Bedeutung, dass ein Ministerium besteht, in welchem die Technik einen Konzentrationspunkt, einen Sammlungspunkt findet; aber noch entscheidender, noch günstiger ist der andere Gesichtspunkt, dass in seiner jetzigen Gestaltung das Ministerium der öffentlichen Arbeiten ein Verkehrs-Ministerium, das Verkehrs-Ministerium ist. “21

Ohne dass damit organisatorische Änderungen verbunden waren, verklammerte und integrierte der programmatisch eingesetzte Verkehrsbegriff die Ressorts Eisenbahn, Wasserbau und Hochbau. Außerdem gab er dem Ministerium der öffentlichen Arbeiten ein modernes und überzeugend zu kommunizierendes Leitbild.

Die Neubestimmung wirkte sich auch auf das Museumsprojekt aus. Komplementär zur Abwehr im parlamentarischen Raum demonstrierte es in der Öffentlichkeit nichts weniger als die Existenzberechtigung des Ministeriums der öffentlichen Arbeiten als Verkehrsministerium in der bestehenden Form. Aus diesem Grund - so vermute ich; auf eine Problematisierung der Umbenennung bin ich nicht gestoßen - wurde der Begriff ,Verkehr“ programmatisch im Namen des geplanten Museums verankert, hier allerdings mit Auswirkungen. Es sollte nicht länger ein Eisenbahnmuseum werden, sondern, die Abteilungen des Ministeriums der öffentlichen Arbeiten spiegelnd, ein „Verkehrs- und Baumuseum“. Die Gründung des Verkehrs- und Baumuseums steht somit in einem direkten Zusammenhang mit den zeitgenössischen, innenpolitischen Diskussionen in Preußen und im Besonderen mit dem umstrittenen Status des Ministeriums der öffentlichen Arbeiten.

\subsection{Demonstration preußischer Identität}

1903 präsentierte Oskar von Miller seine Vision für ein nationales „Museum von Meisterwerken der Naturwissenschaft und Technik“. Auch hier ist anzunehmen, dass Vorbereitungen vorausgingen; auch deshalb der programmatische Titel „Um 1903“ für den Sammelband zu den Anfängen des Deutschen Museums.

Zuerst wurde das Projekt nach außen abgeschottet und diskret vorangetrieben. Von Miller befürchtete die Konkurrenz der Städte Frankfurt und Berlin. 1899 hatte die Stadt Nürnberg das Bayerische Eisenbahnmuseum mit dem Angebot größerer Räumlichkeiten aus München weggelockt. Berlin wäre durchaus auch ,ein idealer Standort gewesen“. ${ }^{22}$ Doch Oskar von Miller hatte sich unwiderruflich für seine Heimatstadt München entschieden.

Nach der Etablierung der Organisations- und Kommunikationsstrukturen wurde die Diskretion zugunsten einer flächendeckenden Netzwerk- und Reklamearbeit aufgegeben. Wirkungsvoll und erfolgreich war diese Arbeit vor

21 Fr. E. (wie Anm. 18), S. 207, Hervorhebung im Orig. gesperrt gedruckt [E. Sch.].

22 Füß1 (wie Anm. 3), S. 265 u. 271. 
allem deshalb, weil sie über eine große Zahl von persönlichen Kontakten und eine umfangreiche Medienberichterstattung vorangetrieben wurde. Die Resonanz war bekanntlich überwältigend und ließ auch Preußen nicht unberührt. Von Miller hatte Verbindung mit dem einflussreichen Verein deutscher Ingenieure VDI aufgenommen und sich der Unterstützung zahlreicher Großindustrieller aus ganz Deutschland versichert; parallel dazu mit dem Bayrischen Königshaus, dieses wiederum mit Kaiser Wilhelm II.

In Preußen beobachtete man sehr genau, was in München vor sich ging, und auch Wilhelm II. hatte mit Datum vom 1. Juli 1903 einen Bericht über den Verein in München angefordert. ${ }^{23}$ Zwei Monate nach der internen Gründungsversammlung in München wendeten sich der preußische Minister der auswärtigen Angelegenheiten von Bülow und der Minister der geistlichen pp. Angelegenheiten von Studt gemeinsam an Kaiser Wilhelm II. Zunächst referierten sie, dass das Projekt „Deutsches Museum von Meisterwerken der Naturwissenschaften und Technik" nicht nur von einem wissenschaftlichen Standpunkt aus zu betrachten sei. Von einem politischen Standpunkt aus gäbe es Bedenken, wonach ,der von partikularistischem Beigeschmack nicht ganz freie Gedanke mitspiele [...], München gewissermaßen zur zweiten deutschen Kapitale zu machen“. Die Unterzeichnenden erklärten sich gegen die Bedenken und dafür, ,daß ein in München zu errichtendes für allgemeine deutsche Zwecke bestimmtes und dabei jeglichen politischen Characters entbehrendes Institut wie das projektierte Museum nicht nur unbedenklich ist, sondern vielmehr ein neues Band zwischen Lagern und dem gemeinsamen deutschen Vaterlande bilden kann und somit dem weiteren Wurzelfassen des Reichsgedankens in Lagern förderlich sein dürfte“. ${ }^{24}$ Die Pointe des Briefs: Die beiden Politiker in Berlin politisierten nun ihrerseits das vermeintlich ,,jeglichen politischen Characters entbehrende [...] Institut" in München, indem sie darin, eher unpreußisch, dafür im Sinne des Kaisers, eine Förderung der Reichsidee erkannten.

Ein Vierteljahr nach dieser Stellungnahme seiner Minister erreichte den Kaiser ein Schreiben auf allerhöchster, d.h. auf seiner Ebene. Als „Protector des Vereins“ zur Unterstützung des Deutschen Museums bat Ludwig Prinz von Bayern: „Eure Kaiserliche und Königliche Majestät möchten den Bestrebungen des für ganz Deutschland wirkenden, bedeutungsvollen Vereins Höchstderen mächtige und gnädigste Unterstützung und Förderung angedeihen zu lassen geruhen.“ ${ }^{25}$ Als umtriebiger Förderer ,ziviler und militärischer Techniken“, wie Wolfgang König in anderen Zusammenhängen aus-

23 Schreiben vom 11. Februar 1904, Berlin, GStAPK: I. HA Rep. 89 Nr. 20517, Bl. 2f. Wann er erstattet wurde, ist unklar.

24 Gezeichnet Bülow, Studt, Berlin 5. Juli 1903, Berlin, GStAPK: I. HA. Rep. 76 V b sekt. 1 Tit. X Nr. 6, Bd. 1, Bl. 7v, 8 .

25 Schreiben vom 21. Oktober 1903, Berlin, GStAPK: I. HA Rep. 89 Nr. 20517, Bl. 4. 
geführt hat, sagte Kaiser Wilhelm II. seine „Unterstützung und Förderung“ offiziell im März 1904 zu. $^{26}$

Wie stellte sich der preußische Minister der öffentlichen Arbeiten, Generalmajor a.D. von Budde, zu dem Projekt? Die Interessen-Kollision liegt auf der Hand:

1. Loyalität gegenüber dem Kaiser, der zugleich König von Preußen war und als Deutscher Kaiser das Projekt in München unterstützte;

2. Von Budde war Minister eines umstrittenen und auf Identitätspräsentation bedachten Ministeriums;

3. als Chef des Reichsamtes für die Verwaltung der Reichseisenbahnen war von Budde der Eisenbahn in Deutschland und in Preußen eng verbunden. ${ }^{27}$

Knapp ein Jahr nach der Gründung des Deutschen Museums, am 21. Juni 1904, schrieb von Budde ebenfalls an Kaiser und König Wilhelm II. Er stellte sich grundsätzlich hinter das Projekt für ein Deutsches Museum. Die Leitidee der in München geplanten Ausstellung sei,

die „historische Entwicklung der naturwissenschaftlichen Forschung, der Technik und der Industrie in ihrer Wechselwirkung darzustellen und ihre wichtigsten Stufen durch hervorragende und typische Meisterwerke zu veranschaulichen. Es soll als deutsche Nationalanstalt Gemeingut aller deutschen Bundesstaaten werden. Nicht nur im deutschnationalen Interesse sondern auch aus dem Grunde halte ich die Förderung des Unternehmens für wünschenswerth, weil besonders auch die Angehörigen der Eisenbahnverwaltung voraussichtlich aus den Sammlungen des Museums reiche, auch für die practische Handhabung des Dienstes werthvolle Belehrung schöpfen werden“". ${ }^{28}$

Bedenkt man, dass zu diesem Zeitpunkt das Bayrische Eisenbahnmuseum bereits von München nach Nürnberg umgezogen war und mehr noch, dass der Ausbildungsgedanke für das Eisenbahnwesen in Preußen ein wesentlicher Bestandteil in der Begründung für das spätere Verkehrs- und Baumuseum werden sollte, bleibt der letzte Zusatz in Bezug auf die Darstellung des Eisenbahnwesens im geplanten Museum der Meisterwerke unklar. Es ist nicht mehr als eine Vermutung, dass von Budde damit vorausschauend einen Anspruch formulierte, den das Deutsche Museum als Nationalanstalt gar nicht würde

26 Schreiben vom 7. März 1904, Berlin, GStAPK: I. HA. Rep. 89 A Nr. 20517, B1. 7.; Wolfgang König, Kaiser - Technik - Krieg. Wilhelm II. und die Förderung ziviler und militärischer Techniken, in: Torsten Meyer u. Marcus Popplow (Hg.), Technik, Arbeit und Umwelt in der Geschichte. Günter Bayerl zum 60. Geburtstag, Münster, New York, München u. Berlin 2006, S. 239-252.

27 Der Minister der öffentlichen Arbeiten von Budde war zugleich oberster Dienstherr des Reichsamts für die Verwaltung der Reichseisenbahnen; während seiner aktiven Militärdienstzeit gehörte er fast 14 Jahre der Eisenbahn-Abtheilung des großen Generalstabes an, die letzten sechs Jahre als ihr Chef; s. Fr. E. (wie Anm. 5), S. 335.

Berlin, GStAPK: I. HA. Rep. 89 Nr. 20517, B1. 9f. 
einlösen können, um so mehr aber das vom eigenen Ministerium geplante „Eisenbahnmuseum“, da das Eisenbahnwesen noch nicht „verreichtlich“, sondern in den Händen der einzelnen deutschen Staaten lag.

Aktuell jedoch gewährte das Ministerium der öffentlichen Arbeiten wie bereits für das Berliner Institut für Meereskunde und für die Kaiser Wilhelm Bibliothek in Posen „Frachtfreiheit“ „während der Zeit der ersten Einrichtung höchstens bis Ende des Jahres 1907““. ${ }^{29}$ Guter Wille war bewiesen. Doch das reichte nicht aus.

Programmatik ist das eine, die Umsetzung ein anderes. Gründungsmuseen benötigen Sammlungen. Mit Umsicht und mit großem Einsatz begann das Deutsche Museum, Wunschlisten für Ausstellungsstücke aus ganz Deutschland zusammenzustellen. Der dabei zutage tretende Anspruch war umfassend. ${ }^{30}$ Dass dabei Schwierigkeiten auftreten würden, war offensichtlich. Deshalb versprach sich Oskar von Miller Vorteile von persönlicher Fürsprache an einflussreicher Stelle, d.h. hierarchisch von ganz oben, vom Kaiser und vom bayrischen König herab bis zu den Unternehmern und Interessenvertretern und bot ihnen gegebenenfalls einen Sitz im Vorstand des Museums an. Spätestens jetzt begann man in Preußen, die damit verbundene Gefahr zu erkennen. Jetzt ging es um mehr als die ideelle Unterstützung und um Frachtfreiheit. Jetzt ging es um die Abgabe von Kulturgut. Emotionen und Pathos kamen ins Spiel.

Am 7. März 1904 war die Zusage Wilhelms II. zur Unterstützung des Münchner Projekts erfolgt. Einen Monat später, am 6. April 1904, schrieb das Königliche Ministerium der auswärtigen Angelegenheit an den Herrn Minister der geistlichen, Unterrichts- und Medizinal-Angelegenheiten von Studt:

„Der hiesige Königlich Bayerische Gesandte hat [...] im Auftrage seiner Regierung davon Mitteilung gemacht, dass der Vorstand des in München gegründeten Museums von Meisterwerken der Nat. u. Technik den Wunsch ausgesprochen hat, es möchte dem Museum zur Ausstellung geeignete Gegenstände seitens der Königlichen Preußischen Regierung überwiesen werden. “31

Mitteilung hierüber erfolgte an die Staatssekretäre des Reichsmarineamts, des Reichspostamts, des Reichsamts des Innern. In der überlieferten Akte folgt dann eine Liste „Notizen, betreffend historische Objekte [...] in Kgl. Preuß. Lehranstalten“ für das Museum von Meisterwerken „von besonderem Wert"; $; 2$ aufgeführt werden historische Objekte mit Bezug auf die mit ihnen verbundenen Männer.

Lag es an der mangelnden Resonanz? Oder gehörten Parallelaktivitäten zur Strategie der Sammler aus München? Warum es knapp ein Jahr später zu

29 Berlin, GStAPK: I. HA. Rep. 89 Nr. 20517, Bl. 10.

30 S. oben Füßl (wie Anm. 4).

31 Berlin, GStAPK: I. HA Rep. 76 V b sekt. 1 Tit. X, Nr. 6, Bd. 1, B1. 73.

32 Aufgezählt wurden die Universitäten Berlin, Bonn, Breslau, Göttingen, Königsberg, die Technische Hochschule Berlin u. die Königliche Bibliothek, Berlin, ebd. 
einer erneuten offiziellen Bemühung um Sammlungsgegenstände aus Preußen kam, ist nicht bekannt. Im Februar 1905 wandten sich die Direktoren des Deutschen Museums, Dr. von Miller, Dr. von Dyck und Dr. von Linde direkt an Minister von Studt, parallel dazu an seinen Ministerialdirektor Dr. Althoff mit der Bitte, ,das mitfolgende Gesuch dem Herrn Staatsminister zur wohlgemeinten Berücksichtigung zu empfehlen“":33

„An den Kgl. Preuss. Minister der geistlichen, Unterrichts- und Medizinalangelegenheiten Hrn. Dr. Studt, Excellenz, Berlin

Euer Excellenz!

In den Lehrmittelsammlungen der wissenschaftlichen Institute finden sich vielfach historische Apparate, welche als Hilfsmittel einer früheren Zeit für Lehrzwecke heute nicht mehr geeignet sind, oft sogar als unliebbarer Ballast angesehen werden und als solcher mitunter in Vergessenheit geraten und im Laufe der Zeit zerstört werden, während gerade solche Gegenstände in hohem Masze würdig wären, als dauernde Zeugen wissenschaftlicher Forschung in einem deutschen Museum für alle Zeiten erhalten zu bleiben.

Als Beispiele solcher historisch wertvoller, für Lehrzwecke aber wenig geeigneter Apparate möchte[n wir] nur eine Originalcamera von Daguerre, eine Hahnsche Rechenmaschine, Modelle historisch interessanter Heissluftmaschinen und dgl. in der Technischen Hochschule zu Charlottenburg erwähnen. Soweit derartige Apparate und Einrichtungen in bayerischen Hochschulen sich befinden, sollen dieselben nach einer gütigen Verfügung des Kgl. Bayerischen Staatsministeriums für Kirchen- und Schulangelegenheiten unserer deutschen Nationalanstalt überwiesen werden.

Euer Excellenz wären wir zum grössten Dank verpflichtet, wenn durch Ihre gütige Entschliessung auch die Kgl. Preussischen Unterrichtsanstalten die Ermächtigung erhielten, derartige, für Lehrzwecke nicht geeignete Apparate unserem Museum zu überweisen, wobei das Eigentums- und Verfügungsrecht dem Kgl. Preuss. Staat selbstverständlich vorbehalten bleiben könnte. “" ${ }^{\text {34 }}$

Wenn sich eine Behörde Zeit nimmt, bedeutet das nicht, dass in der Zwischenzeit nichts passiert. Vier Monate nach dieser Anfrage wandte sich Minister von Studt direkt an den Vertreter des Deutschen Museums von Lerchenfeld und signalisierte grundsätzliches Entgegenkommen. „Inwieweit dies aber möglich sein wird, darüber haben sich in den beteiligten Kreisen erhebliche Meinungsverschiedenheiten geltend gemacht. “" ${ }^{35}$ Die hier mitschwingende Reserve bezog sich offenbar auch auf von Studt selbst. Noch bevor er sich selbst an die entsprechenden Lehranstalten gewandt hatte, so war ihm mitgeteilt worden, hatte sich ,der Museumsvorstand auch unter der Hand mit zahlreichen Institutsdirektoren wegen Abgabe solcher Gegenstände brieflich in

33 Berlin, GStAPK: I. HA Rep. 92 Althoff A I Nr. 216, B1. 4.

34 Berlin, GStAPK: I. HA Rep. 92 Althoff A I Nr. 216, B1. 5f.

35 Schreiben vom 27. Juni 1905, Berlin, GStAPK: I. HA Rep. 76 V b sekt. 1 Tit. X, Nr. 6, Bd. 1, B1. 74. 
Verbindung gesetzt. Es entsteht die Frage, inwieweit es mit den Interessen der Institute des diesseitigen Ressorts, welche sich im Besitze derartiger Gegenstände befinden, vereinbar ist, dass diese letzteren nach München abgegeben werden. Ich ersuche, die Frage wohlwollend zu prüfen“. ${ }^{36}$ Mit diesem Vorspann war die Bitte um wohlwollende Prüfung mehr eine rhetorische Formel aus gebotener Loyalität, als eine engagierte Empfehlung. ${ }^{37}$

Die Reaktionen waren in der Tat erheblich. Sie reichten von aufgeschlossenem Entgegenkommen des Geheimen Regierungsrats und Professors Alois Riedler von der TH Charlottenburg bis zur Absage des Kuratoriums aus Bonn, „dass die Denkmäler einer großen Zeit für das Institut von unschätzbarem Werte" seien; und ,so kann der Direktor nicht einwilligen, jene geheiligten Zeugnisse gewaltiger Geistesarbeit abzugeben". ${ }^{38}$

Rückwirkungen auf die robuste Vorgehensweise des Münchner Museumsvorstands haben sich aus dieser Verstimmung offenbar nicht ergeben. Die Klage eines Sammlers für das Berliner Meeresmuseum aus dem Jahr 1912 fasste die Malaise aus preußischer Sicht zusammen:

„Leider mußte ich die bedauerliche Erfahrung machen, daß uns überall das Deutsche Museum in München zuvorgekommen ist, das alle vorhandenen Modelle an sich genommen hat. Nebenbei darf ich noch erwähnen, dass die Taktik dieses Instituts Beachtung verdient. Das Deutsche Museum hat überall die maßgebenden Herren in den Vorstand oder in Ausschüsse gewählt, um sie noch mehr für die Sache zu interessieren." ${ }^{\text {“39 }}$

Im Hinblick auf die Konkurrenz aus München musste das Ministerium der öffentlichen Arbeiten reagieren. Von Budde machte die im Verborgenen gehegte Idee für ein Verkehrs- und Baumuseum in der Landes- und Reichs-

36 Berlin, GStAPK: I. HA Rep. 92 Althoff A I Nr. 216, B1. 7v.

37 Siehe auch Schreiben vom 27. Juli 1905 von von Studt an die KGl. TH Charlottenburg, an die Universitätskuratoren in Königsberg, Greifswald, Breslau, Halle, Kiel, Göttingen, Marburg, Bonn, Münster, Berlin, an das Physiolog. und an das Physikalische Institut der Rheinischen Friedrich-Wilhelms-Universität, das astrophysikalische Institut in Potsdam und an den Generaldirektor der Königlichen Bibliothek in Berlin, Berlin GStAPK: I. HA Rep. 76 V b sekt. 1 Tit. X, Nr. 6, Bd. 1, B1. 78, 79.

38 Schreiben vom 9. August 1905, ebd., B1. 140v, 141; die Akte enthält mehrere Befürwortungen Riedlers im Zusammenhang mit seinen Überlegungen zu einem kulturgeschichtlichen Konzept anstelle eines Museums der Meisterwerke in München. Wie auch Prof. Intze hatte Prof. Riedler nach Zustimmung von Minister von Studt vom 5.2.1904 einen Sitz im Vorstand des Deutschen Museums erhalten. Riedlers Alternativkonzept wurde in der Literatur bereits ausführlich dargestellt und diskutiert, weshalb hier nicht weiter darauf eingegangen werden braucht; s. dazu: Maria Osietzki, Die Gründungsgeschichte des Deutschen Museums von Meisterwerken der Naturwissenschaften und Technik in München 1903-1906, in: Technikgeschichte 52, 1985, S. 49-75, hier S. 62ff.

39 Zitiert nach Bettina Probst, Das Institut und Museum für Meereskunde - eine bewegte Geschichte? in: Aufgetaucht. Das Museum für Meereskunde im Museum für Verkehr und Technik, Berlin 1996, S. 11-25, hier S. 16; eine Unstimmigkeit in Bezug auf das Datum oder den Empfänger des Briefs konnte noch nicht aufgelöst werden. 
hauptstadt Berlin öffentlich. ${ }^{40}$ Sollte nun etwas aus Bayern angefordert werden, konnte man mit guten Gründen absagen.

Maßgeblich für die Gründung des Verkehrs- und Baumuseums in Berlin waren demnach zwei Ziele: 1) die Demonstration einer institutionellen Identität gegenüber den innenpolitischen Versuchen zur Auflösung des Ministeriums der öffentlichen Arbeiten und 2) die Demonstration staatspatriotischer, preußischer Identität mit der Rettung preußischen Kulturguts vor dem $\mathrm{Zu}$ griff aus dem Ausland Bayern.

\section{Stummer Konsens als Konzept}

Auffällig ist, dass aus der Frühphase des Verkehrs- und Baumuseums, d.h. vor seiner Eröffnung, nichts über Diskussionen zu unterschiedlichen Auffassungen über Sinn und Zweck eines Museums bekannt geworden ist. Dabei betrat, wer sich um 1900 mit Ideen für ein Museum der Technik beschäftigte, kein Neuland. Auf die eine oder andere Weise waren Technik und Industrie in zahlreichen temporären Ausstellungen (Weltausstellungen, Industrie- und Gewerbeausstellungen) und in Museen als ständige Ausstellungen im In- und Ausland vertreten. Konzeptionen und Methoden auf der Grundlage unterschiedlicher Auffassungen zur Geschichte, Kulturgeschichte, Fachausbildung und Volksbildung waren Themen der Museumsreformbewegung um $1900 .{ }^{41}$ Eine instruktive Verortung des Projekts Deutsches Museum in der internationalen Museumsszene aus dem Jahr 1906 stammt von Conrad Matschoss. ${ }^{42}$

Baurat Haas aus dem Ministerium empfahl der Museumskommission die Besichtigung des Postmuseums und der Urania in Berlin „mit Rücksicht auf die dort gebotenen mannigfaltigen Anregungen“, bei einer späteren Besprechung „erläuterte“ der Geheime Ober-Baurat Hossfeld ,anhand von Fotografien und Zeichnungen den Bau und die Einrichtung des Nürnberger Museums“. Inwieweit sich neben den drei Kommissionsvertretern aus dem Ministerium auch die acht Kommissionsvertreter aus den Eisenbahndirektionen bei anderen Museen kundig machten, ist nicht bekannt. In der Ungestörtheit der abgeschirmten Institution scheinen sich die Mitglieder aus der Eisenbahn-

40 O.V., Ein Verkehrs- und Baumuseum in Berlin, in: Zentralblatt der Bauverwaltung 7, 21. Januar 1905, S. 51.

41 Sie bezieht sich allerdings vorwiegend auf die Kunst- und auf die Kulturmuseen. Das „Stilraum-Prinzip“ etwa im Kaiser-Friedrich-Museum (Bode-Museum) war nicht unumstritten, desgleichen die Stil-Collage des Bayerischen Nationalmuseums. S. dazu: Alexis Joachimides, Die Museumsreformbewegung und die Entstehung des modernen Museums 1880-1940, Dresden 2001.

42 Sie steht möglicherweise im Zusammenhang mit seiner Bewerbung um die Stelle als Direktor; s. Conrad Matschoss, Ueber technische Museen, in: Prometheus 840, 1906, S. 124ff.; Eine Übersicht über die naturwissenschaftlichen und technischen Museen ,,in großer Zahl“" erschien in der Zeitschrift Prometheus 16, 1905; s.a. L. Deinhard, Das Deutsche Museum. Seine Entstehung und der in ihm vorhandene Ehrensaal, in: Prometheus 18,1907 , S. $282-285$. 
führungselite auf die Gewissheit ihrer persönlichen Sachkompetenz beschränkt zu haben. Über die Art und Weise, wie ein Museum einzurichten und zu betreiben sei, scheint eine Art stummer Konsens im Anschluss an die Leitideen aus dem Ministerium bestanden zu haben, wie sie von Budde 1905 umriss. Jede Konfrontation vermeidend und damit allseits konsensfähig sollte das Museum „der allgemeinen Besichtigung zugänglich gemacht werden“, „für die Geschichte und Technik des Eisenbahn- und Bauwesens und für die Einrichtungen des Verkehrswesens in weiten Kreisen des Volkes Verständnis und Interesse [...] erwecken“ sowie „Fachleuten, Beamten und Studierenden in mannigfacher Beziehung Belehrendes [...] bieten und ihre Kenntnisse [...] fördern". ${ }^{43}$

In der Allgemeinheit der Hinweise auf Volksbildung, staatliche Selbstrepräsentation, die Herstellung von Akzeptanz in der Bevölkerung sowie die ingenieur-wissenschaftliche Fachausbildung und Weiterbildung spiegelten sich allgemein anerkannte, nicht von Polarisierung gefährdete Gedanken aus den zeitgenössischen Diskursen zur Funktion von Kultur und Technik. Darüber konnte man im Einzelnen diskutieren. Es wurde auch darüber gestritten. Entscheidend für das Ministerium der öffentlichen Arbeiten war jedoch ein anderer Punkt. Um Zustimmung zu erhalten, musste das neue Museum dem preußischen König ohne Anlass zum Konflikt präsentiert werden, denn als deutscher Kaiser unterstützte Wilhelm II. das Deutsche Museum in München und zwar mit großer Anteilnahme.

Diese ebenso komplexe wie delikate Konstellation war den Beteiligten bewusst und konnte wenn, dann nur mit größter Vorsicht problematisiert werden. Dementsprechend stieß ich auch nur auf eine bezeichnende Stellungnahme des Ministers der öffentlichen Arbeiten von Breitenbach, dem Nachfolger von Buddes. Dabei ging es um Oskar von Millers Schachzug, die Vertreter der deutschen Staaten für den Aufsichtsrat des Deutschen Museums zu gewinnen. Minister von Breitenbach:

„Da dieses Museum [in Berlin; Zusatz E. Sch.] auf dem Gebiet des Eisenbahnwesens und des Wasserbaues ähnlichen Zwecken zu dienen bestimmt ist wie das deutsche Museum in München, so war es mir zweifelhaft, ob eine unmittelbare Beteiligung meines Ressorts an dem Aufsichtsrat des letzteren angemessen sein möchte, wenn ich auch gern bereit war und es weiter sein werde, mein Interesse dieser Anstalt zuzuwenden und dies gegebenenfalls auch durch Austausch von Modellen und anderen Schaustücken weiterhin zu betätigen, soweit sich hierzu Gelegenheit und Mittel bieten werden. ${ }^{44}$

Loyalität und Abgrenzung - von Breitenbachs diplomatische Stellungnahme brachte beides zum Ausdruck. Nicht ein irgendwie herausragendes Konzept, sondern die diplomatisch geschickte Positionierung in einem dichten

43 An des Kaisers und Königs Majestät, Berlin, den 20. Januar 1905 (wie Anm. 14).

44 Der Minister für öffentliche Arbeiten Breitenbach, Berlin 3. August 1906, an den Herrn Minister der geistlichen, Unterrichts- und Medicinal-Angelegenheiten, Berlin, GStAPK: I. HA Rep. 76 V b sekt. 1 Tit. X, Nr. 6, Bd. 1, Bl. 227. 
Spannungsfeld - das eine zu wollen, ohne das andere abzulehnen - ließ das Verkehrs- und Baumuseum Wirklichkeit werden. Schließlich benannte von Breitenbach den Geheimen Baurat Haas aus seinem Ministerium. Er sollte die Interessen seines Ministeriums im Aufsichtsrat des Deutschen Museums wahrnehmen.

\section{Sammlungsaktivitäten}

Auch über Aufbau, Struktur und Präsentation der Sammlungen im Detail gelangte vor der Eröffnung des Verkehrs- und Baumuseums nur sehr Allgemeines an die Öffentlichkeit:

„Dabei werden sich die Sammlungen nicht auf rein technische Dinge beschränken, sondern beispielsweise bei dem Eisenbahnwesen auch Gegenstände aus dem Gebiete der allgemeinen Verwaltung, des Tarifs-, Verkehrs-, Abrechnungsund Beförderungswesens, der Personal- und Wohlfahrtsangelegenheiten enthalten. Daher wird dieses Museums nicht allein dem Publikum Gelegenheit geben, die Geschichte, Entwicklung und Technik des Eisenbahn- und Bauwesens kennen zu lernen, sondern es wird auch wegen der Reichhaltigkeit und übersichtlichen Anordnung der Sammlungen den Beamten und Studierenden die Belehrung erleichtern. ${ }^{645}$

Spätestens ab 1904, mit dem Beginn der offiziellen Vorbereitungen für das preußische „Verkehrs- und Baumuseum“, begannen in Berlin wie in München umfangreiche Aktivitäten zum Aufbau und zur Ergänzung der Sammlungsbestände. Seit den 1890er Jahren beschickte der preußische Staat die bedeutenden Industrie-, Welt- und Gewerbeausstellungen in Europa und Amerika mit wertvollen Modellen und anderen Ausstellungsgegenständen (z.B. Chicago 1893; Paris 1900; St. Louis 1904; Mailand 1906). Zu manchen Ausstellungen waren Führer mit Beschreibungen der ausgestellten Gegenstände und mit kurzen geschichtlichen Abrissen erschienen. Das Verkehrs- und Baumuseum bemühte sich um Überlassung oder Ankäufe einzelner Ausstellungsgegenstände.

Was auf diesem Weg nicht beschafft werden konnte, gab die Museumsleitung bei Modellbauern in Auftrag. Zur Eröffnung hatte die Sammlung eine beträchtliche Größe erreicht. 1906 setzte sich der Bestand für die Abteilung Eisenbahn aus 2500 Ausstellungstücken und für die Abteilung Wasser- und Hochbau aus 250 Ausstellungsstücken zusammen. Erneut bestätigt sich, wo das Verkehrs- und Baumuseum seine Wurzeln hatte. Man bezeichnete es nach wie vor „meist kurzweg als ,Eisenbahnmuseum““.46

45 Ein Verkehrs- und Baumuseum in Berlin (wie Anm. 40), S. 51.

46 Brüstlein, Das neue Verkehrs- und Baumuseum in Berlin, in: Zentralblatt der Bauverwaltung, Nr. 1001, 14. Dezember 1906, S. 648ff., hier S. 648; Dementsprechend waren es auch vorwiegend die Eisenbahnzeitschriften, die über das Museum berichteten. In einem Beitrag wurde es gleichsam als Pilgerstätte charakterisiert „,ür alle Jünger des Eisenbahnwesens“" (zitiert nach Brennigk [wie Anm. 1], S. 58). - Immer wieder boten 
Der gravierende Unterschied bei der Exponat-Beschaffung des bürokratisch agierenden Verkehrs- und Baumuseums und des agilen Deutschen Museums: Wo das Deutsche Museum anfragen und bitten musste, ordnete das Ministerium an. Mit Datum vom 31. März 1905 erhielten die zentralen Dienststellen in Preußen einen identischen Brief. ${ }^{47}$ Aufzustellen seien Bestandslisten mit festgelegten Angaben ,nicht nur mit Pläne(n), Modelle(n) pp. von neueren Anlagen, sondern auch auf das Verkehrs- und Bauwesen bezügliche(n) Gegenstände, Karten, Pläne(n), Modelle(n), Bilder(n), Drucksachen usw. von geschichtlicher Bedeutung", und ein Bericht darüber innerhalb von zwei Monaten an das Ministerium der öffentlichen Arbeiten abzugeben, denn ,thunlichste Beschleunigung aller Vorbereitungen“" sei geboten. ${ }^{48}$

Die originäre Domäne des Ministeriums der öffentlichen Arbeiten war der Wasserbau. Sein herausragendes und international beachtetes Projekt war der politisch schwer umkämpfte Mittellandkanal. 1899 hatte man den ersten Abschnitt, den Dortmund-Ems-Kanal, fertig gestellt. Das Schiffs-Hebewerk Henrichenburg repräsentierte diesen Erfolg und trug die Botschaft, dass der Bau des Mittellandkanals fortgesetzt werden würde, weit über Preußen hinaus. Es war zu einem Botschafter von Verkehr, Technik und Politik geworden. Am 1. April 1905 passierte die große Wasservorlage des preußischen Staats das Parlament mit Erfolg. Der Bau des Mittelandkanals konnte fortgesetzt werden. Ein Dokument dieses Erfolgs und des sich damit verknüpfenden Selbstbewusstseins ist der Führer durch die Sammel-Ausstellung aus dem Gebiete des Wasserbaus, veranstaltet vom Königlich Preußischen Ministerium der öffentlichen Arbeiten, Berlin 1906 - kein Führer für das Verkehrs- und Baumuseum, sondern für die Weltausstellung 1906 in Mailand.

Weisungsgemäß stellte auch die Königliche-Kanalbau-Verwaltung in Münster ein Verzeichnis zusammen. Ganz oben auf ihrer Liste stand das Modell des Schiffs-Hebewerks Henrichenburg. ${ }^{49}$ Das war zu erwarten. Etwas Überraschendes enthielt das Münsteraner Verzeichnis aber doch. Das Dokument endet mit einer etwas rätselhaften Passage:

„Zur Erwägung gestellt wird [...] die Aufnahme des der Bauverwaltung gehörigen, von der Gute-Hoffnungshütte gefertigten Modells eines Schiffshebe-

auch Unternehmen dem Museum Schenkungen an. Nicht unerwähnt bleiben sollte die Stiftung des Berliner Unternehmen F. Butzke und Co.: ein Klosett-Hahn mit der wunderbaren Produktbezeichnung „Orkan“.

47 Der Minister der öffentlichen Arbeiten, Berlin, 31. März 1905, an die Oberpräsidenten (Strombau bezw. Kanalverwaltung) in Danzig, Breslau, Magdeburg, Hannover, Münster und Coblenz, sämtliche Regierungs-Präsidenten, Polizeipräsidenten, die Königliche Ministeriale Baukommission in Berlin, den Leiter der Landesanstalt für Gewässerkunde, den Leiter der Versuchsanstalt für Wasserbau und Schiffbau, den Vorsteher des Bureaus für das Hauptnivellement in Berlin, Münster, Nordrhein Westfälisches Staatsarchiv: Bestand Wasser- und Schifffahrtsdirektion West Nr. 976.

Ebd.

49 Undatiertes Konzept; ebd. 
werks mit Parallelsteuerung (System Prüsmann). Das Modell ruht zur Zeit in Kisten verpackt im Geräteschuppen bei der Hanekenschleuse. Seine betriebsfähige Aufstellung würde rd. 8000 M. erfordern. “50

Zur Überwindung einer Gelände-Stufe kannten die Wasserbauer seit mehreren Jahrhunderten die Geneigte Ebene und die Schleuse. Senkrecht-Hebewerke waren eine vergleichsweise junge Entwicklung. ${ }^{51}$ Der Ehrgeiz, das erste Schiffs-Hebewerk für große Binnenschiffe zu bauen, beflügelte gegen Ende des 19. Jahrhunderts viele Ingenieure und Unternehmen in Europa, in Deutschland, in Preußen, unter ihnen den Regierungs-Baumeister Prüsmann aus der Königlichen Wasserbauverwaltung Münster, die für den Bau des Dortmund-Ems-Kanals verantwortlich war. Mit der Gutehoffnungshütte Oberhausen hatte Prüsmann einen interessierten Partner gefunden. 1890 begann die Zusammenarbeit, 1892 präsentierten sie das Ergebnis: eine Denkschrift und ein spektakuläres Großmodell im Maßstab 1:15. Eine hochrangig besetzte Kommission des Ministeriums der öffentlichen Arbeiten begutachtete Modell und Pläne und zeigte sich ,,im Prinzip überzeugt“، 52

Nicht überzeugt war die Privatwirtschaft. Sie protestierte. Es habe keinerlei öffentliche Ausschreibung und damit keine Möglichkeit zur Beteiligung gegeben. Zweifellos hatte das Ministerium der öffentlichen Arbeiten einen schwerwiegenden Fehler gemacht. Es hatte ein Unternehmen bevorteilt. Im letzten Augenblick wurde die Notbremse gezogen. Das Ministerium schrieb einen Wettbewerb aus. Daran beteiligten sich fünf größere Maschinenfabriken und Schiffswerften mit zehn verschiedenen Entwürfen zu SenkrechtHebewerken und geneigten Ebenen. Gewinner war das Unternehmen Haniel \& Lueg. Im Frühjahr 1894 erteilte das Ministerium der öffentlichen Arbeiten ihm den Auftrag zum Bau des Schiffs-Hebewerks Henrichenburg.

Die Gutehoffnungshütte stand in der Reihe der Verlierer. Dort war die Enttäuschung so groß, dass das Unternehmen nicht dazu bereit war, dem Ministerium der öffentlichen Arbeiten das teure Modell für die Weltausstellung in Chicago (1894) zur Verfügung zu stellen. ${ }^{53}$ Das Modell verschwand

50 „Über die Einzelheiten des Modells und die Platzfrage nehme ich Bezug auf den von der Kanal-Kommission erstatteten Bericht vom 3. März 1896 Nr. 21101“; ebd.

51 S. dazu Eckhard Schinkel, Schiffslift. Die Schiffs-Hebewerke der Welt. Menschen - Technik - Geschichte, Essen 2001.

52 Bericht vom 18. März 1892, Münster, Nordrhein Westfälisches Staatsarchiv: Wbd 627. Mitglieder der Kommission waren für das Ministerium der öffentlichen Arbeiten, Berlin, der Civilingenieur Cramer, Schiffbaudirektor Haack, Oberbaudirektor Wiebe, Geh. Baurath Dresel, für die Königliche Kanal-Kommission aus Münster der Reg. und Baurath Oppermann, Reg. Baumeister Prüsmann, für die Gutehoffnungshütte Generaldirektor Ziegler aus Oberhausen, für das Werk Sterkrade der Gutehoffnungshütte Direktor Professor Krohn und Ingenieur Bohse.

53 S. dazu Eckhard Schinkel, Welches Hebewerk sollen wir Bauen? Technik-Entwicklung im Wettbewerb zwischen Staats-Bauwesen und Privat-Wirtschaft, in: Mitteilungen des Canal-Vereins 23, Rendsburg 2003, S. 139-167. 
in Kisten und aus dem Bewusstsein - bis zum Jahr 1905, als die Anforderung aus Berlin kam, Objekte für das neue Museum bereit zu stellen.

Zweifellos war das Modell ein „Hingucker“, und insofern war es nur konsequent, es dem neuen Museum zur Verfügung zu stellen. Es war auch ein wichtiger Baustein - ein „Stadium“ - auf dem Weg der Ingenieure zum ersten Schiffshebewerk der Welt in derartigen Dimensionen. Aber es war auch ein Modell mit einer ungewollten Geschichte. Als offizielles Geschichtszeichen war es gleich auf zweifache Weise ungeeignet: Es verkörperte einen Beinaheskandal des Ministeriums und die peinliche Niederlage eines einflussreichen Unternehmens aus der Privatwirtschaft.

„Verständnis und Interesse zu erwecken“; ,in mannigfacher Beziehung Belehrendes zu bieten": So stand es im Programm des Museums. Nicht dabei stand, dass das Leitbild des Erfolgs, die im Produkt erfolgreich finalisierte Entwicklung die Auswahl und Präsentation des Museums bestimmten. Eine zeitgenössische Formulierung in den Überlegungen der Museumskommission für einen Sammlungskatalog belegt, dass man sich darüber im klaren war. Neben einer ausreichenden Beschreibungen der Objekte müsse er eine Aufzählung der geschichtlichen Daten, Raumabmessungen, Gebrauchsmethoden enthalten, „wobei aber eine Beurteilung der einzelnen Gegenstände derart, dass dadurch Gegenurteile hervorgezogen werden könnten, vermieden ist ${ }^{\text {" }} .{ }^{54}$ Nicht der kulturgeschichtliche Diskurs mit Gegenurteilen, sondern die teleologisch-eindimensionale Entwicklungsgeschichte mit dem vollendeten Ergebnis in der Gegenwart - einem „Meisterwerk“ - war auch eine implizite Leitidee des Berliner Museums.

Eine Reaktion aus Berlin auf das Münsteraner Angebot ist nicht bekannt. Auch bei den Dienststellen der Kanalbauverwaltung hat das Modell nicht überlebt. Vermutlich hätte auch das Münchner Museum der Meisterwerke die Kisten mit dem GHH-Prüsmann-Modell dankend abgelehnt; dafür hätte die grundlegende Überzeugung bestehen müssen, dass zu einer Darstellung von Geschichte auch die „Gegenurteile“ gehören. Ungeachtet der Tatsache, dass im Deutschen Museum die „Meisterwerke“ und ihre ,großen Männer“ gefeiert wurden und sich im Verkehrs- und Baumuseum in Berlin eine Administrations- und Funktions-Elite feierte, bestanden diesbezüglich keine grundlegend unterschiedlichen Auffassungen zwischen ihnen. Als abhängige Geschichtszeichen unterlagen die ausgestellten Gegenstände denselben Rücksichten einer Institution im Aufbau: die Dokumentation von Auseinandersetzungen und Widersprüchen, von Konkurrenzen und Niederlagen war dabei ungewollt.

54 Bemerkungen zur Sitzung der Museumskommission vom 16. März 1908, Berlin, DTM: HH 1 bis 4, Bd. 4. 


\section{Gleichklang und Konkurrenz}

Ein National-Museum für ganz Deutschland in München und ein preußisches Staatsmuseum in der Reichshauptstadt Berlin: ungleiche Geschwister. Beide Institutionen waren auf die Kooperation mit z.T. denselben Institutionen und Personen aus Politik, Wirtschaft und Gesellschaft angewiesen, allen voran Wilhelm II., Deutscher Kaiser und König von Preußen. Interessenkollisionen waren vor allem beim konkreten Interesse an denselben Gegenständen für die aufzubauenden Sammlungen absehbar.

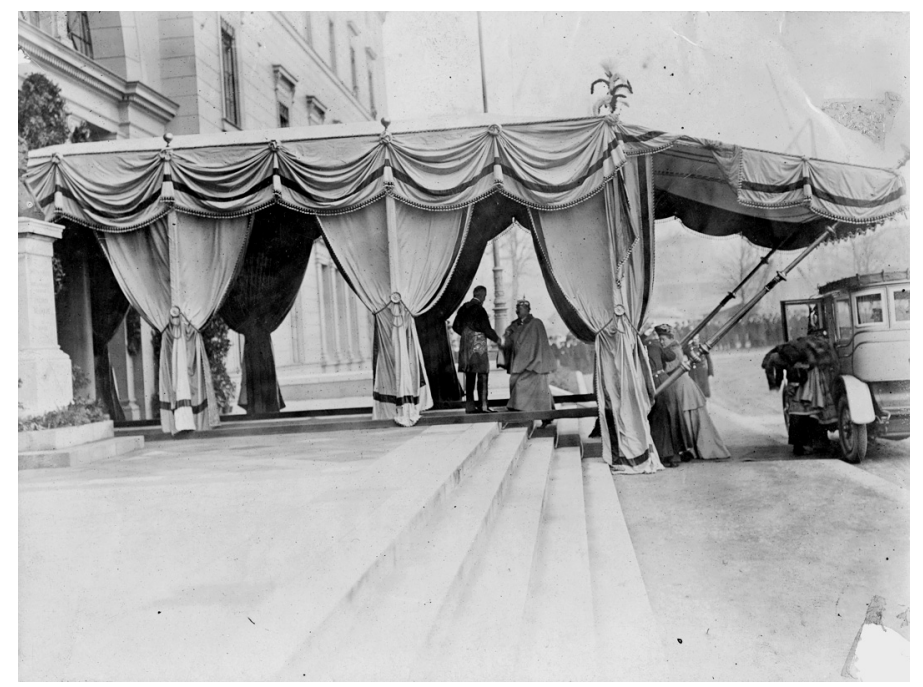

Abb. 2: Eröffnung des Verkehrs- und Baumuseums am 14.12.1906: Minister von Breitenbach begrüßt Kaiser Wilhelm II. Foto: Deutsches Technikmuseum, Berlin.

Zur Eröffnung des Verkehrs- und Baumuseums am 14. Dezember 1906 kam Kaiser Wilhelm II. Ein erlesener Personenkreis stand zu seinem Empfang bereit. Der Minister der öffentlichen Arbeiten, Staatsminister von Breitenbach, hielt eine Ansprache. Anschließend bat er Wilhelm II. um die Eröffnung. Einer Legende zufolge soll der Kaiser nicht einmal gelächelt haben. Geschichtlicher Kontrast und Tiefenschärfe kommen in dieses Eröffnungsszenario, wenn man berücksichtigt, dass Wilhelm II. drei Wochen vorher, am 13. November, in München war. Hier hatte er im Mittelpunkt eines Zeremoniells gestanden, bei dem alle Register bajuvarisch-barocker Prachtentfaltung gezogen worden waren. Anlass und Höhepunkt dieses Besuchs war die offizielle Grundsteinlegung für das Deutsche Museum gewesen. Diesem Ereignis gehörte sein ganzes Wohlwollen und sehr wahrscheinlich auch sein Lächeln.

Ob jemand vom Deutschen Museum bei der Eröffnung des Verkehrsund Baumuseums dabei war? Darüber ist nichts bekannt. Ganz offiziell wollten sich die Münchner das Verkehrs- und Baumuseum erst ein Jahr nach der Eröffnung ansehen. Diese Besichtigung sollte der Schlusspunkt zur Haupt- 
versammlung des Deutschen Museums in Berlin sein, zu der Kaiser Wilhelm II. eingeladen hatte. Zur Vorbereitung des Besuchs schrieb Oskar von Miller an Staatsminister von Breitenbach: „, Für alle Teilnehmer an diesen Versammlungen wäre es von außerordentlichem Interesse, wenn dieselben die mustergiltigen Einrichtungen des königlichen Verkehrs- und Baumuseums besichtigen dürften. “55 Das unterkühlte „mustergiltig“ blieb für sich stehen, ein Bericht von diesem Besuch ist noch nicht aufgetaucht.

Wirft man nun abschließend einen Blick auf die Gründungsgeschichte des Verkehrs- und Baumuseum hier und des Deutschen Museums dort, so findet man in München die kunstvolle Melange aus privatem und privatwirtschaftlichem Engagement und der Einbindung von Kommune, Staat und Reich unter der Regie einer charismatischen, autoritären Gründer-Persönlichkeit, ${ }^{56}$ in Berlin - in krisenhafter Konstellation - die bürokratische Umsetzung einer Staatsinitiative ohne einen namhaften Repräsentanten.

$\mathrm{Zu}$ Beginn war dies eine Geschichte von Konkurrenz und Gleichklang, die Geschichte einer prekären Wahlverwandtschaft. Sieben Jahre später war im Verkehrs- und Baumuseum Gelassenheit eingekehrt. Man verstand sich als „Ergänzung“ zu den bestehenden Museen:

„Das Verkehrs- und Baumuseum in Berlin bildet im Verein mit anderen Verkehrsmuseen, so mit dem Reichspostmuseum in Berlin, dem Königlich Bayrischen Verkehrsmuseum zu Nürnberg, dem Eisenbahnmuseum zu Wien, dem Museum für Meereskunde zu Berlin andererseits eine wertvolle Ergänzung. Dies gilt besonders auch von dem großen hochherzigen Werk des deutschen Museums von Meisterwerken der Naturwissenschaft und Technik in München." ${ }^{\text {"57 }}$

Anschrift des Verfassers: Dr. Eckhard Schinkel, LWL-Industriemuseum, Grubenweg 5, 44388 Dortmund, E-Mail: e.schinkel@1wl.org

55 Die ,von den Bundesstatten [sic], von wissenschaftlichen und technischen Vereinigungen ernannten Mitglieder des Vorstandsrates, sowie die Mitglieder des Ausschusses, welche sich aus den wissenschaftlichen und technischen Kreisen des gesamten deutschen Reiches zusammensetzten“; Schreiben vom 6. November 1907, München, Deutsches Museum, Archiv: VA 0389. 1908 besuchte ein Ingenieur Leonhard Schöner das Verkehrsund Baumuseum zu „Studien“ im Auftrag des Deutschen Museums. Dazu brachte er ein wiederum äußerst förmliches Empfehlungsschreiben Oskar von Millers mit. Der Bericht mit den „gewünschten Aufschlüssen“ ist ebenfalls nicht auffindbar, Oskar von Miller an Reg.baum. Bolstorff, 12. Juni 1908, ebd.: Registratur DM, Ordner 0389.

56 Füß1 (wie Anm. 3).

57 Rechenschaftsbericht der Museumsverwaltung v. März 1913, S. 8f. (wie Anm. 9). 
Impressum

Wissenschaftliche Leitung:

Gerhard Dohrn-van Rossum

Wolfgang König

Reinhold Reith

Karin Zachmann

Redaktion:

Dr. Martina Blum, Dr. Katharina Zeitz
Herausgeber: Verein Deutscher Ingenieure (VDI)

Gesellschaft für Technikgeschichte (GTG)

Frühere Wissenschaftliche Leitung:

Friedrich Klemm (1965-1975)

Karl-Heinz Ludwig (1976-1997)

Conrad Matschoß (1909-1941)

Kurt Mauel (1974-1994)

Wilhelm Treue (1965-1992)

Ulrich Troitzsch (1976-2000)

Adam Wandruszka (1965-1969)

Ulrich Wengenroth (1995-2006)

Die Zeitschrift TECHNIKGESCHICHTE schließt nach einem Registerband 31 (1965) mit dem Band 32 an das Jahrbuch Technikgeschichte, Beiträge zur Geschichte der Technik und Industrie, Bd. 1 bis 30 (1909 bis 1941), an.

TECHNIKGESCHICHTE veröffentlicht Beiträge über die geschichtliche Entwicklung der Technik in ihren wissenschaftlichen, gesellschaftlichen, wirtschaftlichen und politischen Zusammenhängen. Die Aufsätze dieser Zeitschrift werden begutachtet (refereed journal).

Anschrift der Schriftleitung:

Dr. Martina Blum, c/o Deutsches Museum Museumsinsel 1, D-80538 München

Tel. 089 / 2179405 Fax 089/2179 408

E-Mail schriftleitung.technikgeschichte @mzwtg.mwn.de

\section{Anschrift der Redaktion:}

Dr. Katharina Zeitz, Technische Universität Berlin, Institut für Philosophie, Sekr. H 72 Straße des 17. Juni 135, D-10623 Berlin Tel. 030/31424085 Fax 030/31425962 E-Mail Technikgeschichte@TU-Berlin.de

Anschrift des Verlags:

edition sigma e.Kfm.

Karl-Marx-Str. 17, D-12043 Berlin

Tel. 030 / 6232363 Fax 030 / 6239393

E-Mailverlag@edition-sigma.de

\section{Hinweise für Autor/inn/en:}

Beachten Sie bitte die Hinweise für die Manuskripteinreichung in diesem Heft. Die Zusendung von Büchern zur Rezension an die Schriftleitung ist willkommen, Rezensionsexemplare können jedoch nicht zurückgesandt werden.

Anzeigenverwaltung: edition sigma (Anschrift siehe oben). Zurzeit gilt Anzeigentarif 1/2007

Druck: Rosch-Buch GmbH, Scheßlitz Gedruckt auf umweltfreundlichem, alterungsbeständigem Papier.

Printed in Germany.

\section{Bezugsbedingungen:}

Jahresabonnement (4 Hefte): 78,00 Euro; für Studierende*: 46,80 Euro. - Einzelheft: 21,50 Euro. - Vorzugspreis* für Mitglieder des Vereins Deutscher Ingenieure, der Deutschen Gesellschaft für Geschichte der Medizin, Naturwissenschaft und Technik und der GeorgAgricola-Gesellschaft: Jahresabo 70,20 Euro, Einzelheft 19,50 Euro. (* Studien- bzw. Mitgliedschaftsnachweis erforderlich.) - Alle Preisangaben: zuzügl. Versandkosten ab Verlagsort; einschl. gesetzl. Mehrwertsteuer. - Für Mitglieder der Gesellschaft für Technikgeschichte ist der Bezug der Zeitschrift im Beitrag enthalten.

Abonnements verlängern sich um jeweils ein Jahr, es sei denn, sie werden spätestens sechs Wochen vor Ende des Bezugszeitraums schriftlich beim Verlag gekündigt. - Die zur Verwaltung von Abonnements erforderlichen Datenwerden beim Verlag unter Beachtung der Vorschriften des Bundesdatenschutzgesetzes elektronisch gespeichert.

Copyright: edition sigma e.Kfm.

Alle in dieser Zeitschrift veröffentlichten Beiträge sind urheberrechtlich geschützt. Kein Teil dieser Zeitschrift darf außerhalb der engen Grenzen des Urheberrechtsgesetzes ohne vorherige schriftliche Zustimmung des Verlags reproduziert, übersetzt oder verbreitet werden.

TECHNIKGESCHICHTE im Internet: 